\title{
MORe PREcISE: A Multicenter Prospective Cohort of Patient Reported Outcome Measures in Stroke
}

\author{
Amber E Corrigan ( $\sim$ a.elyse.corrigan@gmail.com ) \\ Department of Biostatistics and Health Informatics, Institute of Psychiatry, Psychology \& Neuroscience, King's College London, London \\ Ben Carter \\ Department of Biostatistics and Health Informatics, Institute of Psychiatry, Psychology \& Neuroscience, King's College London, London \\ Alexander Smith \\ Division of Population Medicine, School of Medicine, Cardiff University, Cardiff, \\ Anna Pennington \\ Aneurin Bevan University Health Board \\ Jonathan Hewitt \\ Division of Population Medicine, School of Medicine, Cardiff University, Cardiff,
}

\section{Research Article}

Keywords: Morbidity, Patient Reported Outcome. PROM. Stroke

Posted Date: September 16th, 2021

DOI: https://doi.org/10.21203/rs.3.rs-832600/v1

License: (c) (i) This work is licensed under a Creative Commons Attribution 4.0 International License. Read Full License 


\section{Abstract}

Background and Purpose: The use of patient reported outcomes measures (PROMs) may offer utility and provide outcome measures that are important for stroke survivors. This study used a stroke specific PROM, which contains Mental health (MH) and Physical Health (PH). The primary aim of this study was to assess the association between the $\mathrm{MH}$ and $\mathrm{PH}$ measures following a stroke and preexisting health conditions.

Methods: A multicenter prospective cohort study at 19 hospital sites across England and Wales during 2019 was conducted. Prevalence of morbidity within PROMs was estimated with 95\% confidence intervals (95\% Cl). The association between each PROM domain and demographic and health conditions were calculated using a multilevel multivariable linear model fitting the adjusted mean difference (aMD).

Results: PROM morbidity was high post stroke; $93.2 \%$ of the participants reported combined post stroke PROM morbidity. The MH domain was associated with pre-existing: diabetes, previous stroke, age, and sex. The $\mathrm{PH}$ domain was found to be associated with gender (female) and previous stroke,

Conclusions: Stroke survivors ubiquitously suffer morbidity. Risk factors associated with worsening MH and PH morbidity have implications in clinical management as they offer an opportunity to intervene, particularly to prevent long term mental health. PROMs provide useful outcome measure that matter to stroke survivors.

\section{Introduction}

The World Health Organisation (WHO) estimates 15 million people suffer from stroke each year: with more than 5 million living with permanent disability ${ }^{1-3}$.Neurological deficits that persist secondary to stroke, is heterogeneous and varies across the patient population.

The use of patient reported outcomes measures (PROMs), defined as patient questionnaires measuring views on health status from the perspective of the patient, rather than the clinician, are growing in importance and significance. They provide alternative patient facing outcome measures, that actually matter to patients ${ }^{4}$. PROMs offer a manner in which to measure specific functional domains in a way which is meaningful to the patient and is able to encapsulate the patient's own perspective on their health ${ }^{5}$. To improve the use of PROMs within the stroke community, a consensus Stroke Standard Set of outcome data has been developed which promotes the use of patient reported outcomes as part of a valuebased assessment of care ${ }^{4}$.

\section{Aims}

The aim of this study was to determine the prevalence of PROM morbidity and further the association between PROM and clinical outcomes and stroke risk factors.

\section{Methods}

\section{Study design}

The full study protocol has previously been published 6 . In brief, this cohort of survivors were recruited between the period of August 2018 and October 2019 from 19 hospital sites with acute and hyper-acute patient facilities in the UK. Baseline data was assessed within 14 days of poststroke event (herein defined as the baseline period post-stroke), with a 3 and 6 month follow up.

The COVID-19 pandemic impacted on the study follow up and herein report baseline data in the acute stroke setting. Data collection was conducted by trained and experienced research staff.

\section{Ethical approval}

All participates provided the informed consent to participate for the study. All methods were conducted in accordance with relevant guidelines and regulations. Ethical approval was granted by the NHS Research Ethics Committee - Wales REC 3 - 18/WA/0299 - Health and Care Research Wales Support and Delivery Centre for all the sites

\section{Measures}

Demographic, lifestyle and clinical measures

During the baseline assessment the following were assessed: age; sex; stroke type; pre stroke smoking; alcohol consumption; level of care: clinical characteristics which included past medical history (hypertension, diabetes; transient ischemic attacks and prior stroke).

Patient-Reported Outcome Measures (PROM) 
A Stroke Specific PROM, developed by Salinas et a/ ${ }^{4}$ was used. It contains 10 questions from the Patient-Reported Outcomes Measurement Information System Global Health Short Form-10 (PROMIS-10), which has two domains of physical health (PH) and mental health (MH). This is a validated and established patient-reported outcome measure ${ }^{4,7-8}$. Salinas et al added an additional 5 stroke specific questions.

Short-Form Montreal Cognitive Assessment (SF-MoCA)

The SF-MoCA is an adapted shorter 10-point version of the 30 item Montreal Cognitive

Assessment. This contains three sections, comprising of clock drawing, abstraction and 5-word recall This tool may act as an indicator of poststroke cognitive impairment, utilising a threshold score of 7 or below ${ }^{9}$.

Patient Health Questionnaire-9 (PHQ-9)

The PHQ-9 $9^{10}$ is a primary care screening tool for depression and has previously been recommended in stroke with strong psychometric properties ${ }^{11}$.The recommended cut off used in stroke, and the one utilised in this study in terms of morbidity is above or equal to $8^{11}$.

\section{Generalised Anxiety Disorder-7 (GAD-7)}

Whilst the GAD- $7^{12}$ has not been validated within stroke use, it is a wildy used screening tool for generalised anxiety in primary care, a stroke specific a threshold of above or equal to 5 was determined ${ }^{13,14}$.

Modified Rankin Scale (mRS)

The $\mathrm{mRS}^{15}$ is delineated using the Rankin Focussed Assessment (RFA), a questionnaire that allows global consideration of disability after the occurrence of stroke. Questions from the RFA are scored on the Modified Rankin scale from 0-5, with 0 being the lowest level of disability and 5 being the highest ${ }^{16}$.

\section{Data Analysis}

All data analysis were undertaken in Stata version 16.0. The measures were scored using the validated methods and missing item missingness (e.g. no more than $30 \%$ ) within each measure (or domain) within participant were pro-rata mean imputed ${ }^{17}$. Participants with over $30 \%$ of missing items were scored as missing.

Prevalence of morbidity within PROM measures

In order to quantify morbidity within the PROMIS 10, morbidity was defined across each domain as a supra-threshold response to the clinical threshold set for the total PROMIS 10 response (a score of 85 and below to indicate morbidity ${ }^{5-8,18}$ ). This was at the acute baseline period. $95 \%$ confidence intervals $(95 \% \mathrm{Cl})$ were reported. Domain specific analysis was conducted for the cognitive and physical domains of the PROMIS 10 .

\section{Outcomes}

The co-primary outcomes were the MH and PH domains. Secondary outcomes included the GAD-7; PHQ-9; mRS, SF-MoCA and the additional 5 stroke specific questions (walking, toileting, dressing, tube feeding and communication).

\section{Covariates}

The following were fitted to assess any association with the outcomes: pre-stroke hypertension, previous TIA, previous stroke, pre-stroke diabetes, male sex, and age.

\section{Statistical Analysis}

The association between exposures and outcomes were fitted using a crude and multivariable multilevel linear model, where hospital site was fitted as a random effect. The multivariable model was adjusted for: age, sex, pre-stroke hypertension, previous stroke event, previous TIA and pre-stroke diabetes diagnosis. The analysis presented the mean difference (MD) and adjusted mean difference (aMD) reported with associated 95\% Cl and $\mathrm{P}$ values.

\section{Results}

\section{Study population}

From the 19 hospitals 550 participants consented into the study, and one subject withdrew prior to completing the baseline visit assessments, leaving 549. The population consisted of 232 women (42.3\%) aged between 25 to 97 (mean=72.7, SD=0.55) (Supplementary Table 1). 
Post-stroke PROM morbidity was in $93.2 \%$ participants. PROM MH was poor in $3.9 \%$ participants was very good in $29.3 \%$, or excellent in $19.1 \%$, and within $\mathrm{PH}$ was with poor in $39.9 \%$, and in very good $7.31 \%$, and excellent in $1.19 \%$ (Table 1). There were 253 (50.9\%) of people were able to walk unaided, 214 (41.4\%) who needed help going to the toilet, 220 (42.6\%) who needed aid to dress, 31 (6.2\%) who needed a tube for feeding and 97 (17.9\%) who had problems communicating or understanding (see Supplementary Table 2).

Demographic and clinical characteristics associated with PROM

Multivariable analysis for $\mathrm{MH}$ was associated with pre-existing diabetes (aMD=-2.01; 95\% Cl $-3.91,-0.12 ; \mathrm{p}=0.036, \mathrm{Table} 2)$; sex $(\mathrm{aMD}=1.91 ; 95 \% \mathrm{Cl}$ $0.28,3.54 ; \mathrm{p}=0.022)$; history of stroke $(\mathrm{aMD}=-3.62 ; 95 \% \mathrm{Cl}-5.86,-1.39 ; \mathrm{p}=0.001)$; and age (aMD=0.07; 95\% Cl: $0.01,0.14 ; \mathrm{p}=0.037)$. $\mathrm{PH}$ were associated with sex $(\mathrm{aMD}=2.09 ; 95 \% \mathrm{Cl} 0.54,3.65 ; \mathrm{p}=0.008)$, and history of stroke $(\mathrm{aMD}=-3.05 ; 95 \% \mathrm{Cl}-5.17,-0.93 ; \mathrm{p}=0.005)$. The other comparisons were not significant $(p>0.05)$.

\section{Demographic and clinical characteristics associated with Clinical Outcomes}

Generalised anxiety using GAD-7 was not found to be associated with any demographic or clinical characteristics ( $p>0.05)$ (Supplementary Table 3). Depression using PHQ9 was associated with pre-stroke diabetes $(\mathrm{aMD}=2.34 ; 95 \% \mathrm{Cl} 1.17,3.50 ; \mathrm{p}<0.001) \operatorname{sex}(\mathrm{aMD}=-1.27 ; 95 \% \mathrm{Cl}-2.28,-0.27$; $\mathrm{p}=0.013$ ), and age 0 . Patients with diabetes had increased depression, male exhibited lower depression, and increasing age had lower depression (Supplementary Table 4).

The $\mathrm{mRS}$ was associated with sex $(\mathrm{aMD}=-0.2695 \% \mathrm{Cl}-0.48,-0.04 ; \mathrm{p}=0.018)$ and age $(\mathrm{aMD}=0.01 ; 95 \% \mathrm{Cl} 0.01,0.02 ; \mathrm{p}=0.003)$. (Supplementary Table 5). The SF-MoCA was found to be associated with pre-stroke hypertension (aMD $=-0.62 ; 95 \% \mathrm{Cl}-1.09,-0.15 ; p=0.009)$, pre-stroke diabetes $(\mathrm{aMD}=-0.59 ; 95 \% \mathrm{Cl}-1.15,-0.03 ; \mathrm{p}=0.039)$ and age $(\mathrm{aMD}=-0.03 ; 95 \% \mathrm{Cl}-0.06,-0.02 ; \mathrm{p}<0.001)$ (Supplementary Table 6).

\section{Discussion}

We included 549 stroke survivors and found morbidity was ubiquitous. MH was associated with age, female sex, previous stroke and pre-stroke diabetes and $\mathrm{PH}$ with female sex and previous stroke. The stroke specific questions ${ }^{4}$ demonstrated that stroke survivors had a high degree of comorbidity.

The PROMIS 10 has been shown as a feasible instrument in stroke survivors ${ }^{19-24}$ and exhibited the components of patient reported morbidity. Particularly when considering the high post-stroke prevalence of PROM morbidity immediately after stroke. The PROM represents an outcome measure that is real and accessible to the stroke survivor. Work by Phillipp et al also demonstrate the instrument across different populations ${ }^{32}$.

Further, the PROM aligns with clinical risk factors of stroke. A pre-stroke diagnosis of diabetes is significantly associated with worse MH PROM outcome scores following a stroke. This is mirrored within the association with other outcome measures; pre-stroke diabetes and a worse MoCA and PHQ9 scores across the cohort at the baseline period (Supplementary Table 3,5). A diagnosis of diabetes is considered a high-risk factor for stroke and confers worse outcomes in terms of overall morbidity, functional outcomes and readmission or recurrence ${ }^{25}$. Diabetes, as a comorbidity, has been accepted as being highly related to overall outcome $\mathrm{e}^{26,27}$ and the reflection within the PROM measures is not surprising.

The study demonstrated that gender was associated with different outcomes, when using the PROM as an outcome measure. Male sex was associated with better cognitive and physical domain scores post-stroke (Table 2 and 3 ). This correlated with the modified Rankin scale, Supplementary Table 3), where male sex to be associated with better physical functioning. This is consistent, with the literature; worse post-stroke outcomes have been previously reported across female patients ${ }^{28-30}$. One large scale study of post stroke outcomes across over 19.000 people reported that 3-6 months after stroke women are more likely to experience disability and worse quality of life ${ }^{31}$.

This study offers a novel insight to the use of a PROMIS 10 to assess post-stroke morbidity and quality of life from the patient perspective. We demonstrate the large prevalence of patient reported morbidity and highlight important clinical and demographic associations for worse patient reported outcomes, notably previous stroke, sex, and pre-stroke diabetes. This adds value for the use of PROMS in clinical practice - demonstrating that poorer PROM outcomes can be predicted by certain, pre stroke factors. By using this stroke specific PROM in the clinical setting, clinicians my potentially enact a more sensitive measure of morbidity, especially as perceived by the stroke survivor.

This was a large UK wide prospective multicentre study that assessed patient reported outcomes. The limitations to our study are that PROMIS 10 may only be useful in patients with mild to moderate impairment ${ }^{32,33}$. While 146 of our subjects (26.9\%) reported post-stroke aphasia, the degree and severity of impairment was not noted. Thus is unclear if the functional impairment in communication may hinder the completion of the PROMIS-10. The use of patient reported tools in patients that struggle with communication is current limitation of all self-reporting measures and should continue to be discreetly considered.

The PROMs offers a standardised and validated manner of patient centred consideration within morbidity estimation; this is with excellent discrimination being shown between the likes of the PROMIS 10 item and component scores and other clinical stroke measurements, such as the 
modified Rankin scale score (mRS) ${ }^{22}$. The best manner and tool to address the disparity across patient and clinician reporting needs further investigation. PROMs such as the PROMIS 10 offers a clinically feasible way to measure patient reported quality of life. In the future, comparison to other generic instruments, such HL-QoL and other generic measures that have been utilised in stroke to acess patient quality of life, such as the SF- $36^{34}$ and EQ- $5 D^{35}$, is needed.

\section{Conclusion}

The PROMs offers a standardised and validated manner of patient centred consideration within morbidity estimation; this is with excellent discrimination being shown between the likes of the PROMIS 10 item and component scores and other clinical stroke measurements, such as the modified Rankin scale score $(\mathrm{mRS})^{22}$. The best manner and tool to address the disparity across patient and clinician reporting needs further investigation. PROMs such as the PROMIS 10 offers a clinically feasible way to measure patient reported quality of life, that may capture a greater understanding of the patient experience in the acute stroke setting. This offers a clinical measure to recognise and measure patient experience. Given that we demonstrate morbidity is significantly higher in PROM measure, than clinical counter parts, in the acute stroke setting, this suggests that the patient experience is not being captured fully. Thus, utilising PROMs in clinical practice would allow greater understanding and reaction to patient perceptions and needs. In the future, comparison to other generic instruments, such HL-QoL and other generic measures that have been utilised in stroke to assess patient quality of life, such as the SF- $36^{34}$ and EQ-5D ${ }^{35}$, is needed.

The high prevalence of morbidity within patient reported outcome measures, and congruence in outcome risk factors across clinical measures, signifies the need to acknowledge and utilise such tools within clinical practice, post-stroke management and as outcome measures within clinical trials that have meaning for patients.

\section{Declarations}

Ethics approval and consent to participate :

All participates provided the informed consent to participate for the study. All methods were conducted in accordance with relevant guidelines and regulations. Ethical approval was granted by the NHS Research Ethics Committee - Wales REC 3 - 18/WA/0299 - Health and Care Research Wales Support and Delivery Centre for all the sites

\section{Consent for publication}

All participates were consented to participate and for publication.

\section{Availability of data and materials}

The datasets used and/or analysed during the current study are available from the corresponding author on reasonable request.

\section{Competing interests}

$\mathrm{JH}$ is funded by the Stroke Research and Innovation Fund (SRIF) of the Stroke Implementation Group (SIG) [Welsh Government] - 03 ABUHB. AS is funded by the Stroke Association (SA PGF 18\100029 Stroke Association Post-Graduate Fellowship).

AC, BC, AP declare no funding associated with this study.

No other non-financial interests.

\section{Contribution of Authors}

JH conceived the study and received funding for the study. AP, AS, BC, and JH designed the study. AP was the study coordinator supported by AS. $\mathrm{AC}$ and $\mathrm{BC}$ developed the statistical analysis plan. Data analysis and interpretation was carried out by $\mathrm{AC}$ and $\mathrm{BC}$. The first draft of the manuscript was carried out by $\mathrm{AC}, \mathrm{BC}$ and $\mathrm{JH}$. All authors have drafted the final manuscript.

\section{Acknowledgements}

We would like to acknowledgements the More Precise study team - consisting of Alexander Smith, Anna Pennington, Ben Carter and Jonathan Hewitt.

We would further like to acknowledge the lead site investigations at each hospital site; Louise Coombe, Sarah Jones, Jayne Thomas, Sharon Storton, Richard Dewar, Lindsay Tarasconi, Benjamin Jelly, Walee Sayeed, Maureen Bartley, Kerry Smith, Sarah Dunne, Sarah Board, Katherine Ahlquist, Angela Kulendran, Ravneeta Singh, Asis Kumar, Deborah Ward, Rachel Teal, Ken Fotherby and Radim Licenik. 


\section{References}

1. Randhawa, H., Pearce, G., Hepton, R., Wong, J., Zidane, I. and Ma, X., 2019. An investigation into the design of a device to treat haemorrhagic stroke. Proceedings of the Institution of Mechanical Engineers, Part H: Journal of Engineering in Medicine, p.095441191989069.

2. King, D., Wittenberg, R., Patel, A., Quayyum, Z., Berdunov, V. and Knapp, M., 2020. The future incidence, prevalence and costs of stroke in the UK. Age and Ageing, 49(2), pp.277-282.

3. Donkor, E., 2018. Stroke in the21stCentury: A Snapshot of the Burden, Epidemiology, and Quality of Life. Stroke Research and Treatment, 2018, pp.1-10.

4. Martins, S., Norrving, B., Salinas, J., Sprinkhuizen, S. and Schwamm, L., 2015. Defining an international standard set of patient-centered outcome measures after stroke. Journal of the Neurological Sciences, 357, p.e394.

5. Reeves, M., Lisabeth, L., Williams, L., Katzan, I., Kapral, M., Deutsch, A. and Prvu-Bettger, J., 2018. Patient-Reported Outcome Measures (PROMs) for Acute Stroke: Rationale, Methods and Future Directions. Stroke, 49(6), pp.1549-1556.

6. Smith, A., Bains, N., Copeland, L., Pennington, A., Carter, B. and Hewitt, J., 2020. Morbidity Prevalence Estimate at 6 Months Following a Stroke: Protocol for a Cohort Study. JMIR Research Protocols, 9(6), p.e15851

7. Hays, R., Schalet, B., Spritzer, K. and Cella, D., 2017. Two-item PROMIS® global physical and mental health scales. Journal of Patient-Reported Outcomes, 1(1).

8. Hays, R., Spritzer, K., Thompson, W. and Cella, D., 2015. U.S. General Population Estimate for “Excellent” to "Poor" Self-Rated Health Item. Journal of General Internal Medicine, 30(10), pp.1511-1516.

9. Mai, L., Oczkowski, W., Mackenzie, G., Shuster, A., Wasielesky, L., Franchetto, A., Patlas, M. and Sahlas, D., 2013. Screening for Cognitive Impairment in a Stroke Prevention Clinic Using the MoCA. Canadian Journal of Neurological Sciences / Journal Canadien des Sciences Neurologiques, 40(2), pp.192-197.

10. Kroenke, K. and Spitzer, R., 2002. The PHQ-9: A New Depression Diagnostic and Severity Measure. Psychiatric Annals, 32(9), pp.509-515.

11. Turner, A., Hambridge, J., White, J., Carter, G., Clover, K., Nelson, L. and Hackett, M., 2012. Depression Screening in Stroke. Stroke, 43(4), pp.1000-1005

12. Spitzer, R., Kroenke, K., Williams, J. and Löwe, B., 2006. A Brief Measure for Assessing Generalized Anxiety Disorder. Archives of Internal Medicine, 166(10), p.1092.

13. Morris, R., Eccles, A., Ryan, B. and Kneebone, I., 2017. Prevalence of anxiety in people with aphasia after stroke. Aphasiology, 31(12), pp.14101415.

14. Kneebone, I., Fife-Schaw, C., Lincoln, N. and Harder, H., 2016. A study of the validity and the reliability of the Geriatric Anxiety Inventory in screening for anxiety after stroke in older inpatients. Clinical Rehabilitation, 30(12), pp.1220-1228.

15. Bloch, R., 1988. Interobserver agreement for the assessment of handicap in stroke patients. Stroke, 19(11), pp.1448-1448

16. Saver, J., Filip, B., Hamilton, S., Yanes, A., Craig, S., Cho, M., Conwit, R. and Starkman, S., 2010. Improving the Reliability of Stroke Disability Grading in Clinical Trials and Clinical Practice. Stroke, 41(5), pp.992-995.

17. Brain, K., Carter, B., Lifford, K., Burke, O., Devaraj, A., Baldwin, D., Duffy, S. and Field, J., 2017. Impact of low-dose CT screening on smoking cessation among high-risk participants in the UK Lung Cancer Screening Trial. Thorax, 72(10), pp.912-918.

18. Carter, B., Hayes, C., Smith, A., Pennington, A., Price, M., Pearson, O., Vitoratou, S. and Hewitt, J., 2021. A single patient reported outcome measure for acquired brain injury, multiple sclerosis \& Parkinson's disease. PLOS ONE, 16(6), p.e0251484.

19. Lam, K. and Kwa, V., 2018. Validity of the PROMIS-10 Global Health assessed by telephone and on paper in minor stroke and transient ischaemic attack in the Netherlands. BMJ Open, 8(7), p.e019919.

20. Rimmele, D., Lebherz, L., Frese, M., Appelbohm, H., Bartz, H., Kriston, L., Gerloff, C., Härter, M. and Thomalla, G., 2019. Outcome evaluation by patient reported outcome measures in stroke clinical practice (EPOS) protocol for a prospective observation and implementation study. Neurological Research and Practice, 1(1).

21. Alonso, J., Bartlett, S., Rose, M., Aaronson, N., Chaplin, J., Efficace, F., Leplège, A., LU, A., Tulsky, D., Raat, H., Ravens-Sieberer, U., Revicki, D., Terwee, C., Valderas, J., Cella, D. and Forrest, C., 2013. The case for an international patient-reported outcomes measurement information system (PROMIS $\left.{ }^{\circ}\right)$ initiative. Health and Quality of Life Outcomes, 11(1),

22. Katzan, I. and Lapin, B., 2018. PROMIS GH (Patient-Reported Outcomes Measurement Information System Global Health) Scale in Stroke. Stroke, 49(1), pp.147-154.

23. . - Reeves, M., Lisabeth, L., Williams, L., Katzan, I., Kapral, M., Deutsch, A. and Prvu-Bettger, J., 2018. Patient-Reported Outcome Measures (PROMs) for Acute Stroke: Rationale, Methods and Future Directions. Stroke, 49(6), pp.1549-1556.

24. Katzan, I., Thompson, N., Lapin, B. and Uchino, K., 2017. Added Value of Patient-Reported Outcome Measures in Stroke Clinical Practice. Journal of the American Heart Association, 6(7). 
25. Lau, L., Lew, J., Borschmann, K., Thijs, V. and Ekinci, E., 2018. Prevalence of diabetes and its effects on stroke outcomes: A meta-analysis and literature review. Journal of Diabetes Investigation, 10(3), pp.780-792.

26. Zsuga, J., Gesztelyi, R., Kemeny-Beke, A., Fekete, K., Mihalka, L., Adrienn, S., Kardos, L., Csiba, L. and Bereczki, D., 2012. Different effect of hyperglycemia on stroke outcome in non-diabetic and diabetic patients - a cohort study. Neurological Research, 34(1), pp.72-79.

27. Piernik-Yoder, B. and Ketchum, N., 2013. Rehabilitation Outcomes of Stroke Patients With and Without Diabetes. Archives of Physical Medicine and Rehabilitation, 94(8), pp.1508-1512.

28. Niewada, M., Kobayashi, A., Sandercock, P., Kamiński, B. and Członkowska, A., 2005. Influence of Gender on Baseline Features and Clinical Outcomes among 17,370 Patients with Confirmed Ischaemic Stroke in the International Stroke Trial. Neuroepidemiology, 24(3), pp.123-128.

29. Gall, S., Phan, H., Madsen, T., Reeves, M., Rist, P., Jimenez, M., Lichtman, J., Dong, L. and Lisabeth, L., 2018. Focused Update of Sex Differences in Patient Reported Outcome Measures After Stroke. Stroke, 49(3), pp.531-535.

30. Reeves, M., Bushnell, C., Howard, G., Gargano, J., Duncan, P., Lynch, G., Khatiwoda, A. and Lisabeth, L., 2008. Sex differences in stroke: epidemiology, clinical presentation, medical care, and outcomes. The Lancet Neurology, 7(10), pp.915-926.

31. Carcel C, Wang X, Sandset E, Delcourt C, Arima H, Lindley R et al. Sex differences in treatment and outcome after stroke. Neurology. 2019;93(24):e2170-e2180.

32. Philipp, R., Lebherz, L., Thomalla, G., Härter, M., Appelbohm, H., Frese, M. and Kriston, L., 2021. Psychometric properties of a patient-reported outcome set in acute stroke patients. Brain and Behavior,.

33. Salter, K., Moses, M., Foley, N. and Teasell, R., 2008. Health-related quality of life after stroke: what are we measuring?. International Journal of Rehabilitation Research, 31(2), pp.111

34. de Haan R. Measuring Quality of Life After Stroke Using the SF-36. Stroke. 2002;33(5):1176-1177.

35. Golicki D, Niewada M, Buczek J, Karlinska A, Kobayashi A, Janssen M et al. Validity of the Eq-5d-5I in Stroke Patients. Value in Health. 2014;17(7):A570.

\section{Tables}

Table 1 - Domain specific breakdown of PRO - Breakdown of Cognitive and Physical domain of the 10 Question PRO across sub-groups of patients. Sub-groups encompass age groups (18-49, 50-85, and above, not including 85), gender (male, female) and patients who report pre-stroke hypertension, previous TIA, previous stroke and pre-stroke diabetes. Results are reported as poor, fair, good, very good and excellent. Domain specific cut offs as delineated by Hays, Spritzer, Thompson and Cella, 2015; Hays, Schalet, Spritzer and Cella, 2017

Table 2: Mean difference of hypertension, TIA, previous stroke, diabetes, sex and age associated with PRO mental health domain. The mean difference (MD) and adjusted MD are reported with associated $p$-values and intervals. Statistically significant $p$-values are reported in bold. As a negative score is associated with worse outcome - a negative value indicates a factor resulting in worse outcome.

Table 3: : Association of hypertension, TIA, previous stroke, diabetes, sex and age on PRO physical health domain. Both crude and adjusted results are reported with associated $p$-values and intervals. Statistically significant $p$-values are reported in bold. As a negative score is associated with worse outcome - a negative value indicates a factor resulting in worse outcome.

\section{Supplementary Files}

This is a list of supplementary files associated with this preprint. Click to download.

- SupplementaryMaterialssubmission.docx 
Table of morbidity outcome domains within PRO Scoring across age, sex and pre-stroke conditions (Hypertension, TIA, stroke and diabetes)

Mental Health domain

Poor Fair Good Very Excellent Poor Fair Good Very Excellent

\section{Age}

$18-49$

$\begin{array}{llll}1(3.2 \%) & 8 & 8 & 8 \\ & (25.8 \%) & (25.8 \%) & (25.8 \%)\end{array}$

$6(19.4 \%)$

$12(38.7$

50-85

18
$(4.7 \%)$

82

(19.9\%)

115

117

81(

$85+$

$1(1.52 \%)$

11
$(16.7 \%)$

(27.7\%) (28.4\%)

$19.6 \%)$

162

(39.2\%) (24.7\%)

29
$(45.3 \%)$

Sex

Female

10

Male

10
$(4.8 \%)$

50

$\begin{array}{lllll}10 & 56 & 81 & 90 & 63(21 \%)\end{array}$

$\begin{array}{llll}10 & 56 & 81 & 90 \\ (3.3 \%) & (18.7 \%) & (27 \%) & (30 \%)\end{array}$

$\begin{array}{lllll}\begin{array}{l}\text { Pre-Stroke } \\ \text { Hypertension }\end{array} & 9(3.3 \%) & 58 & 73 & 78 \\ & & (21.5 \%) & (27.1 \%) & (28.9 \%)\end{array}$

$(21.5 \%) \quad(27.1 \%) \quad(28.9 \%)$

$52(19.3 \%) \quad 100$

88

$24.7 \%)$

$(27.8 \%) \quad(6.8 \%)$

Previous TIA $3(3.6 \%)$

$\begin{array}{lllllllllll} & & (24.1 \%) & (34.9 \%) & (22.8 \%) & (14.5 \%) & (43.2 \%) & (25.3 \%) & (28.9 \%) & (3.6 \%) & \\ \text { Previous } & 8 & 16 & 22 & 23( & 8(10.4 \%) & 37 & 25 & 12 & 1 \\ \text { stroke } & (10.39 \%) & (20.7 \%) & (28.6 \%) & 29.8 \%) & & (48.7 \%) & (32.89 \%) & (15.8 \%) & (1.3 \%) & 1(1.3 \%) \\ \begin{array}{l}\text { Pre-Stroke } \\ \text { Diabetes }\end{array} & 6(4.9 \%) & 30 & 38 & 36 & 13 & 54 & 32 & 26 & 5 \\ \end{array}$

PRO Mental Health domain Mean differences

\begin{tabular}{lllllll} 
& MD & P-value & $(95 \% \mathrm{Cl})$ & Adjusted MD & P-value & $(95 \% \mathrm{Cl})$ \\
\hline Pre-Stroke Hypertension & 0.24 & 0.768 & $(-1.36,1.84)$ & 0.65 & 0.435 & $(-0.97,2.27)$ \\
\hline Previous TIA & -2.01 & 0.067 & $(-4.16,0.14)$ & -1.67 & 0.130 & $(-3.84,0.49)$ \\
\hline Previous stroke & -3.75 & $\mathbf{0 . 0 0 1}$ & $(-5.95,-1.56)$ & -3.62 & $\mathbf{0 . 0 0 1}$ & $(-5.86,-1.39)$ \\
\hline Pre-Stroke Diabetes & -2.20 & $\mathbf{0 . 0 2 2}$ & $(-4.09,-0.31)$ & -2.01 & $\mathbf{0 . 0 3 6}$ & $(-3.91,-0.12)$ \\
\hline Sex (Male) & 1.64 & $\mathbf{0 . 0 4 8}$ & $(0.01,3.25)$ & 1.91 & $\mathbf{0 . 0 2 2}$ & $(0.28,3.54)$ \\
\hline Age & 0.06 & 0.060 & $(-0.01,0.12)$ & 0.07 & $\mathbf{0 . 0 3 7}$ & $(0.01,0.14)$
\end{tabular}

PRO physical health domain Mean differences

\begin{tabular}{lllllll} 
& MD & P-value & $(95 \%$ Cl $)$ & Adjusted MD & P-value & $(95 \%$ Cl $)$ \\
\hline Pre-Stroke Hypertension & -0.22 & 0.774 & $(-1.72,1.28)$ & 0.19 & 0.808 & $(-1.34,1.72)$ \\
\hline Previous TIA & -1.65 & 0.111 & $(-3.67,0.38)$ & -1.25 & 0.234 & $(-3.31,0.81)$ \\
\hline Previous stroke & -3.17 & $\mathbf{0 . 0 0 3}$ & $(-5.27,-1.08)$ & -3.05 & $\mathbf{0 . 0 0 5}$ & $(-5.17,-0.93)$ \\
\hline Pre-Stroke Diabetes & -1.64 & 0.071 & $(-3.44,0.134)$ & -1.48 & 0.107 & $(-3.27,0.32)$ \\
\hline Sex (Male) & 2.02 & $\mathbf{0 . 0 0 9}$ & $(0.50,3.54)$ & 2.09 & $\mathbf{0 . 0 0 8}$ & $(0.54,3.65)$ \\
\hline Age & 0.01 & 0.724 & $(-0.05,0.07)$ & 0.02 & 0.472 & $(-0.04,0.09)$
\end{tabular}

